\title{
1 Peak shifts and extinction under sex-specific selection
}

3 Stephen P. De Lisle

4

5 Evolutionary Ecology Unit, Department of Biology

6 Lund University

$7 \quad$ Sölvegatan 3722362

8 Lund, Sweden

9

10 Email: stephen.de lisle@,biol.lu.se

11

12 Keywords: adaptive landscape, sexual dimorphism, stabilizing selection, microevolution,

13 macroevolution 


\section{Abstract}

27 A well-known property of sexual selection combined with a cross sex genetic correlation

$28\left(r_{\mathrm{mf}}\right)$, is that it can facilitate a peak shift on the adaptive landscape. How do these diversifying

29 effects of sexual selection $+r_{\mathrm{mf}}$ balance with the constraints imposed by such sexual

30 antagonism, to affect macroevolution of sexual dimorphism? Here, I extend existing

31 quantitative genetic models of evolution on complex adaptive landscapes. Beyond recovering

32 classical predictions for the conditions promoting a peak shift, I show that when $r_{\mathrm{mf}}$ is

33 moderate to strong, relatively weak sexual selection is required to induce a peak shift in

34 males only. Increasing the strength of sexual selection leads to a sexually-concordant peak

35 shift, suggesting that macroevolutionary rates of sexual dimorphism may be largely

36 decoupled from the strength of within-population sexual selection. Accounting explicitly for

37 demography further reveals that sex-specific peak shifts may be more likely to be successful

38 than concordant shifts in the face of extinction, especially when natural selection is strong.

39 An overarching conclusion is that macroevolutionary patterns of sexual dimorphism are

40 unlikely to be readily explained by within-population estimates of selection or constraint

41 alone. 


\section{Introduction}

52 A longstanding dilemma in evolutionary biology lies in understanding how populations can

53 evolve from one phenotypic optimum to another. When a population is under net-stabilizing

54 selection and in the vicinity of the optimum trait value (a 'peak'), selection will pull the

55 population towards the nearby optimum (1), leaving alternative optima seemingly

56 inaccessible (2). For a peak shift to occur, some force must allow a population mean

57 phenotype to transcend the pull of the nearby optimum, and cross a fitness valley to climb a

58 peak beyond (3-6).

Candidate phenomena that may facilitate crossing a natural-selection valley include genetic drift, a change in the environment, or sexual selection. Wright famously proposed a key role for drift in valley crossing $(3,5,6)$, although drift alone will only facilitate such a crossing with exceptional rarity, requiring very weak selection (a shallow valley) and small population size (7-9). A change in the environment seems a likely explanation, although also an incomplete one as rates of phenotypic macroevolution do not seem to be coupled to environmental upheaval in any obvious way $(10,11)$. Finally, sexual selection can readily pull a population off a natural-selection fitness optimum (12), resulting in a peak shift even across quite deep valleys (13). If only one sex is under significant sexual selection, whether or not a peak shift occurs in the other sex depends on the magnitude of the cross sex genetic correlation $\left(r_{\mathrm{mf}}\right)$ for the trait (13). If $r_{\mathrm{mf}}$ is high enough, sexual selection in one sex will pull

70 both sexes off of their optimum, leading to a peak shift in both sexes. More generally,

71 directional selection on any trait can induce a peak shift in other, genetically correlated traits that themselves reside on an optimum (9). In this way, sexual selection coupled with crosssex genetic correlations has been proposed as a likely mechanism facilitating peak shifts and thus promoting the origin of diversity (14). 
Two open questions remain in light of sexual selection's likely role in driving peak

77 dimorphism, when adaptive landscapes are complex; That is, when sexually dimorphic and sexually-monomorphic trait optima exist, what are the conditions that promote or constrain likelihood of a peak shift to each optimum? Second, how do we reconcile the diversifying effects of sexual selection and $r_{\mathrm{mf}}$ with the constraining effects that $r_{\mathrm{mf}}$ is expected to have on male and female adaptation? Put another way, although we know sexual selection $+r_{\mathrm{mf}}$ can lead to a peak shift, we also know that this condition of sexual conflict constrains adaptation. In this note I extend Lande's (12) model of sexual and natural selection towards a single optimum to the case of multiple optima. This model is similar to one analyzed by Lande and Kirkpatrick (13), but is agnostic to female preference evolution, a major focus of their model. My analysis reveals two underappreciated features of peak shift models. First, large regions of parameter space exist in which relatively weak sexual selection is required to induce a sex-specific peak shift; strong sexual selection is expected to lead to evolution along a line of sexual monomorphism at the macroevolutionary scale. Second, the latter type of peak shift is severely limited by extinction. Although these effects are consistent with results of previous work $(9,13)$, to my knowledge they have rarely been appreciated, particularly in the context of macroevolution of sexual dimorphism.

\section{The Model - Two-sex 'Twin Peaks'} sexual selection, in which natural selection favors a single optimum value for the male trait $z$ and female trait $y$. His model of natural selection corresponds to a single adaptive peak on

98 the two-sex adaptive landscape (where $\bar{W}=\bar{W}_{m} \bar{W}_{f}$, Figure S1A). Directional sexual selection acts independently of natural selection to redistribute fitness across individuals 
100

101

102

103

104

105

106

107

108

109

110

111

112

113

114

115

116 can define $\beta_{m}$ under the twin peak model in equation 1 as: Peak' model, illustrated in Figure S1C and Figure 1.

where $\theta_{z, 1}$ and $\theta_{z, 2}$ are two phenotypic optima, $P_{z}$ is the phenotypic variance, and $\omega_{z, 1} \omega_{z, 2}$ are the strengths of stabilizing selection (assumed equal throughout). Such an adaptive landscape corresponds to an individual fitness surface (16) with two separate optima. This model is bimodal, with optima in the vicinity of $\theta$, for $2 \theta^{2}>\omega+\mathrm{P}$. The adaptive

landscape for a scenario where only one sex has multiple optima is illustrated in Figure S1B.

If instead we assume females also have multiple optima, using an expression analogous to equation 1 but relating $\bar{W}_{f}$ to the female mean trait value $\bar{y}$, we obtain the 'Two-sex Twin

Given $\beta_{m}=\nabla_{m} \ln \bar{W}_{m}+c_{m}(12)$, where $\nabla_{m}$ is the gradient $\partial / \partial \bar{z}$ and $\mathrm{c}_{\mathrm{m}}$ is sexual selection arising from some mating bias independent of the strength of natural selection, we

$121 \quad \beta_{m}=\bar{W}_{m}^{-1}\left[2 \frac{\left(\theta_{z, 1}-\bar{z}\right)}{\left(\omega_{z, 1}+\mathrm{P}_{z}\right)} \exp \left\{-\frac{\left(\theta_{z, 1}-\bar{z}\right)^{2}}{\left(\omega_{z, 1}+\mathrm{P}_{z}\right)}\right\}+2 \frac{\left(\theta_{z, 2}-\bar{z}\right)}{\left(\omega_{z, 2}+\mathrm{P}_{z}\right)} \exp \left\{-\frac{\left(\theta_{z, 2}-\bar{z}\right)^{2}}{\left(\omega_{z, 2}+\mathrm{P}_{z}\right)}\right\}\right]+c_{m}$ 
123 With an analogous expression for $\beta_{f}$. For simplicity and consistency with past work, I focus

124 on the scenario of sexual selection in males only $\left(c_{f}=0, c_{m} \geq 0\right)$. Evolution of the male

125 and female mean phenotypes, $\bar{z}$ and $\bar{y}$, depends not only on selection within each sex but also

126 on correlated response to selection in the other sex mediated by the cross sex genetic

127 correlation $r_{m f}$ :

$$
\begin{aligned}
& \Delta \bar{z}=\frac{1}{2} \beta_{m} G_{m}+\frac{1}{2} \beta_{f} B \\
& \Delta \bar{y}=\frac{1}{2} \beta_{f} G_{f}+\frac{1}{2} \beta_{m} B
\end{aligned}
$$

128 where $G_{m}$ and $G_{f}$ are the male and female genetic variances and $r_{m f}=B / \sqrt{ }\left(G_{m} G_{f}\right)$.

\section{Population growth}

Population growth can be considered a function of mean fitness, such that

maladaptation carries demographic cost. Following Lande (12), we can describe change in census population size $\mathrm{N}$ as

$$
N_{t+1}=k \bar{W}_{m} \bar{W}_{f} N_{t}
$$

134 where $k$ is constant or function defining per capita birth rates. For simplicity, I assume $k$ as a constant corresponding to density-independent population growth. In equation 4, adaptation in both sexes contributes to population growth, corresponding to a biological scenario where,

137 for example, parental care is shared across the sexes. Alternatively, in many species male

138 adaptation may contribute little to population growth rates, and we can instead define change 139 in census size as

$$
N_{t+1}=k \bar{W}_{f} N_{t}
$$

140 which assumes that there are always at least enough males to fertilize females in the

141 population. Equations 4 and 5 represent two ends of a continuum of in which male

142 adaptation may contribute to population growth rates. 
145 The role of random genetic drift has been considered extensively in peak shift models, and I

146 address it only briefly here for completeness. The rate of evolution by genetic drift will be

147 proportional to $\mathbf{G} / N_{e}(17)$, and in a two-sex population,

148

$$
N_{e}=\frac{4 N_{m} N_{f}}{N_{m}+N_{f}}
$$

149 (18). Drift occurs after natural selection such that each sex is represented proportional to

150 their own mean fitness,

$$
N_{m} \propto \frac{N}{2} \bar{W}_{m}, \quad N_{f} \propto \frac{N}{2} \bar{W}_{f}
$$

152

where $\mathrm{N}$ represents the number of individual (e.g., zygotes) before selection, with N/2 of each sex under a Fisherian primary sex ratio (1). ancestral condition of sexual monomorphism, each simulation was started with the population mean at a sexually-monomorphic optimum of $\bar{z}=\bar{y}=\theta_{z, 2}=\theta_{y, 2}=-2$, with

$159 \theta_{z, 1}=\theta_{y, 1}=2$. In this scenario there are three unoccupied optima, two of which are accessible deterministically under positive sexual selection and $r_{m f}$; one new optimum is along a line of male-female isometry, and represents a shift from one sexually monomorphic peak to another (henceforth, 'sexually-monomorphic', or concordant, peak shift). A second accessible peak involves a male-only shift, and so represents a 'sexually dimorphic' peak 
164

165

166

167

shift. All $\omega$ were assumed equal, and phenotypic variance was set at unity and $G=P / 2$, assumed constant. Starting N was set to 10,000; population growth was assumed as in equation 3 or 4 , although population size was capped at 10,000 with an arbitrary extinction threshold of $\mathrm{N}=20$. The growth constant $k$ was assumed 1.05. Thus growth was exponential and density independent up to the upper bound. Assuming unbounded growth, changing population size, $k$, or genetic parameters did not change qualitative conclusions. Complete $\mathrm{R}$ script is provided as supplemental material.

\section{Results}

When there is only a single optimum, sexual and natural selection jointly determine the equilibrium trait value for males, which are displaced from their peak proportional to sexual selection $\mathrm{c}_{\mathrm{m}}$ and the strength of stabilizing selection $\omega$ (Figure $\mathrm{S} 1 \mathrm{D} ; 12$ ). Female equilibrium trait values are unaffected by the strength of sexual selection in males, but their path towards their optimum is affected by $r_{m f}$ (Figure S1D). When multiple optima exist, the combination of sexual selection and $r_{m f}$ determines if a peak shift occurs and which alternative optima is reached. Two scenarios are shown in Figure 1, illustrating that increasing the strength of sexual selection while holding $r_{m f}$ constant, and vice versa, have similar effects. Unlike the single optimum case, when multiple optima exist, female equilibrium trait values depend on sexual selection in males and $r_{m f}$; for a peak shift to occur in females, their product must be high enough for female mean phenotype to be displaced beyond the critical value required for a peak shift. Note that a 'monomorphic peak' shift (Figure 1A) still entails some sexual dimorphism in the traits will be observed at equilibrium (because males will be displaced optimum), although the equilibrium magnitude of sexual dimorphism will be far higher when a peak shift is restricted to males only (a 'dimorphic 
shift'; Figure 1). Valley crossing carries substantial demographic costs, illustrated in Figure

$\mathrm{S} 2$.

Whether a peak shift occurs for males depends only on the strength of sexual

191 selection $\mathrm{c}_{\mathrm{m}}$. However, whether the shift is dimorphic (males only) or monomorphic depends

192 also on $r_{m f}$, such that for wide ranges of moderate to strong genetic correlations, increasing

193 the strength of sexual selection while holding $r_{m f}$ constant yields first a transition from no

194 peak shift to a dimorphic shift, and then to a monomorphic shift, and then in some cases extinction (Figure 2, left panels). Increasing the strength of stabilizing selection (smaller $\omega$ ) exacerbates these effects. These effects hold regardless of the demographic model assumed, although extinction is far more likely to be observed when population growth rates are a

198 function of both male and female adaptation (Figure 2, right panels). These conclusions remain qualitatively unchanged when accounting for genetic drift, although the outcomes become probabilistic due to the stochastic nature of drift in the fitness valley (Figure S3).

201

\section{Discussion}

203 Although the broad factors that may possibly affect peak shifts are well understood, how these factors jointly determine the probability of actual peak shifts remains a puzzle (6). Sexspecific selection, in particular sexual selection, provides one example of the uncertainties around peak shifts. Sexual selection and genetic correlations may be key in facilitating peak shifts $(12,13)$, yet this diversifying effect (14) is seemingly at odds with the constraining role that such a condition must also manifest $(12,19)$. The point of this paper is to explore how sexual selection and $r_{\mathrm{mf}}$ interact to influence the likelihood of a peak shift occurring, as well as which alternative optima are most likely to be reached. This modest revisit to classical

211 peak shift models yields two underappreciated phenomenon that have important implications

212 for the evolution of sexual dimorphism. 
214 peak shift, whether that peak shift results in substantial sexual dimorphism depends weaker sexual selection produces a peak shift in males only; increasing sexual selection

217 further increases the correlated response in females to the point where females also shift 218 peaks (see also figure 4A in Price et al. 1993). The implication is that when sexually monomorphic optima exist, strong sexual selection is expected to lead to peak shifts along a line of male-female isometry when $r_{\mathrm{mf}}$ is non-zero. Only when sexual selection is weak enough in magnitude that its interaction with $r_{\mathrm{mf}}$ fails to push females beyond the critical displacement required for a peak shift, yet strong enough to directly push males beyond their own critical displacement, will dimorphic optima be reached. This suggests that macroevolutionary patterns of sexual dimorphism may depend far more on nuances of the two-sex adaptive landscape than on the magnitude of within-population sexual selection.

Second, the demographic cost of valley crossing affects which peaks can be reached. When population growth rates depend on adaptation in both sexes, or when natural selection is strong, sex-specific peak shifts are more likely to occur successfully in the face of extinction than are concordant ('monomorphic') shifts. This demographic effect is the result of higher demographic costs for crossing a valley in both sexes. When population growth rates depend only on female adaptation, valley crossing in males-only carries no demographic cost, and so may be more likely to be observed. These effects are amplified under strong selection (deep valleys), and indicate that extinction-generated survivorship bias (20) may make sex-specific (dimorphic) peak shifts more likely to be observed than monomorphic shifts. observed in macroevolutionary studies of sexual dimorphism. First, a large number of 
studies have investigated the link between proxies for the strength of within-population

241 dimorphism (21-24). This finding of little correlation between the magnitude (yet some correlation between the sign; 25) of sexual dimorphism and sexual selection proxies is consistent with the results presented here. Concomitantly, any association between $r_{\mathrm{mf}}$ and

$26,27)$ could have important macroevolutionary consequences. varying distance from each other, will surely be more complex than the model presented here. None the less, whether a peak shift to a dimorphic or monomorphic peak occurs will depend in part on an interaction between sexual selection and genetic correlations that may decouple the strength of sexual selection from the magnitude of resulting sexual dimorphism at equilibrium, and crossing two valleys at once may be demographically costly.

Acknowledgements I thank David Punzalan, Locke Rowe, Erik Svensson, Beatriz Willink, 
A

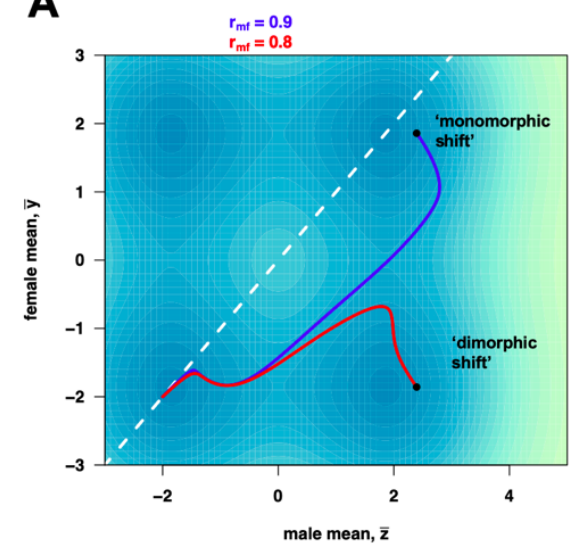

C

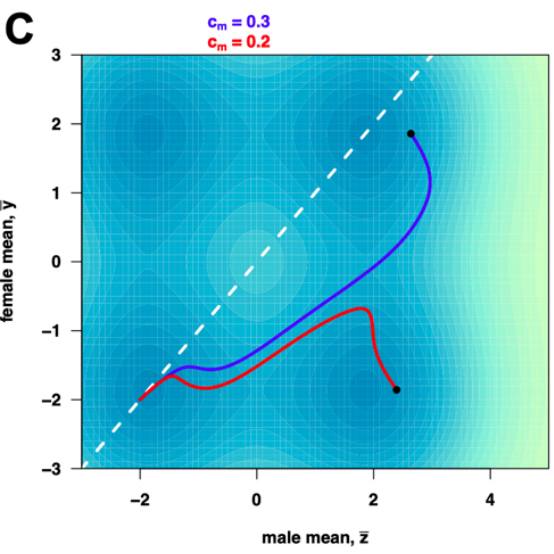

B

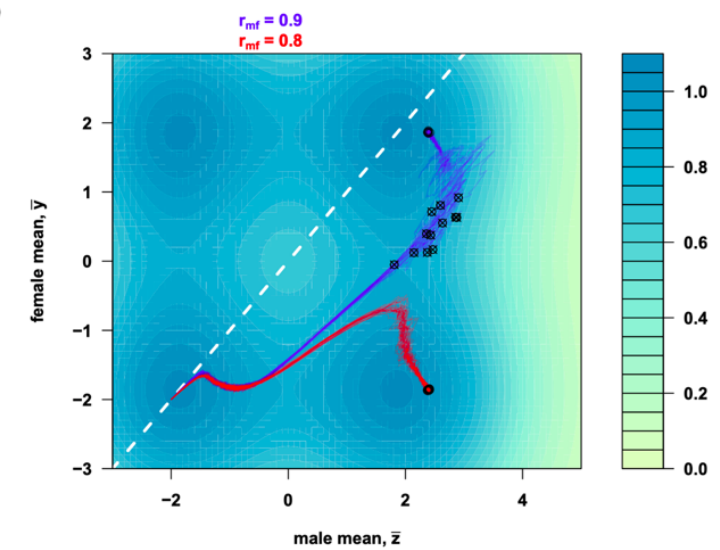

D

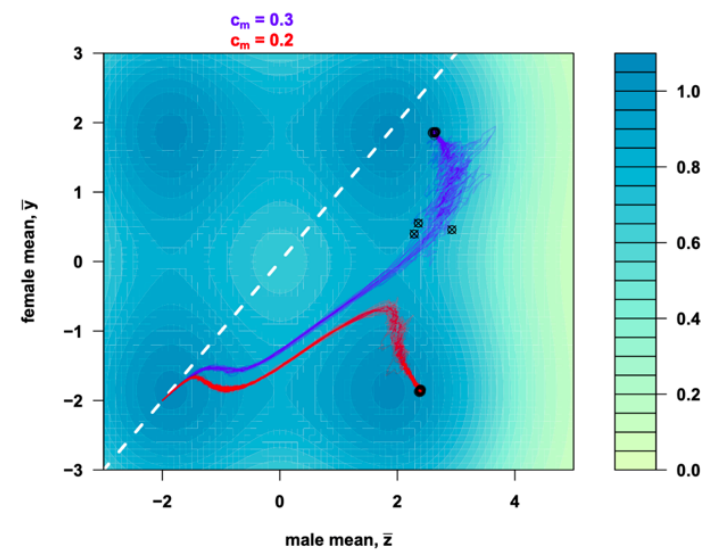

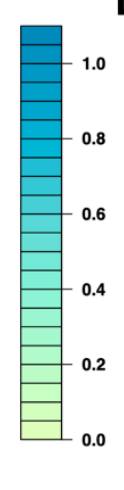

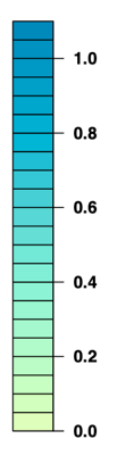

Figure 1. Peak shifts induced by sexual selection in the Two-sex Twin Peaks model.

Panel A shows two deterministic trajectories, starting from the lower left optimum,

corresponding to two different values of the cross-sex genetic correlation $r_{\mathrm{mf}}$. Although

sexual selection is strong enough to induce a peak shift, which new optimum reached depends upon the value of $r_{\mathrm{mf}}$. Panel B illustrates 20 replicates of stochastic evolution (i.e., with drift) under the same parameter values as A and assuming female demographic dominance $\left(N_{t+1}=k \bar{W}_{f} N_{t}\right)$. Round circles illustrate the mean values after 10000

271 generations of evolution; extinction events are denoted with a crossed circle. Panel $\mathbf{C}$ contrasts trajectories under two strengths of sexual selection $\mathrm{c}_{\mathrm{m}}$, with otherwise identical parameter values. Panel D illustrates stochastic evolution under the same parameter values as C. White dashed line illustrates the line of sexual monomorphism, for reference. In A and B, $\mathrm{c}_{\mathrm{m}}=0.2 ; \mathrm{B}$ and $\mathrm{C} r_{\mathrm{mf}}=0.8$; other parameter values as described in text. 


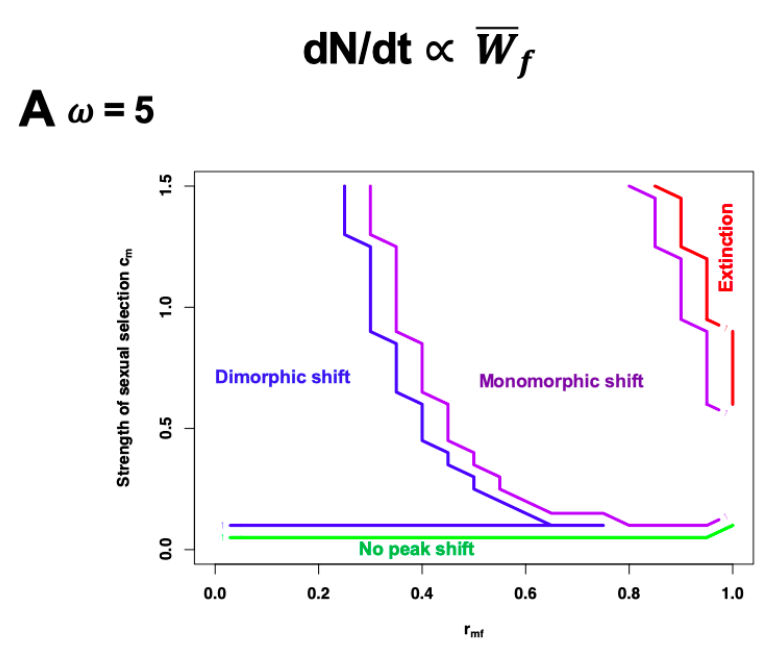

$\mathrm{dN} / \mathrm{dt} \propto \bar{W}_{\boldsymbol{f}} \bar{W}_{m}$

B $\omega=3.5$
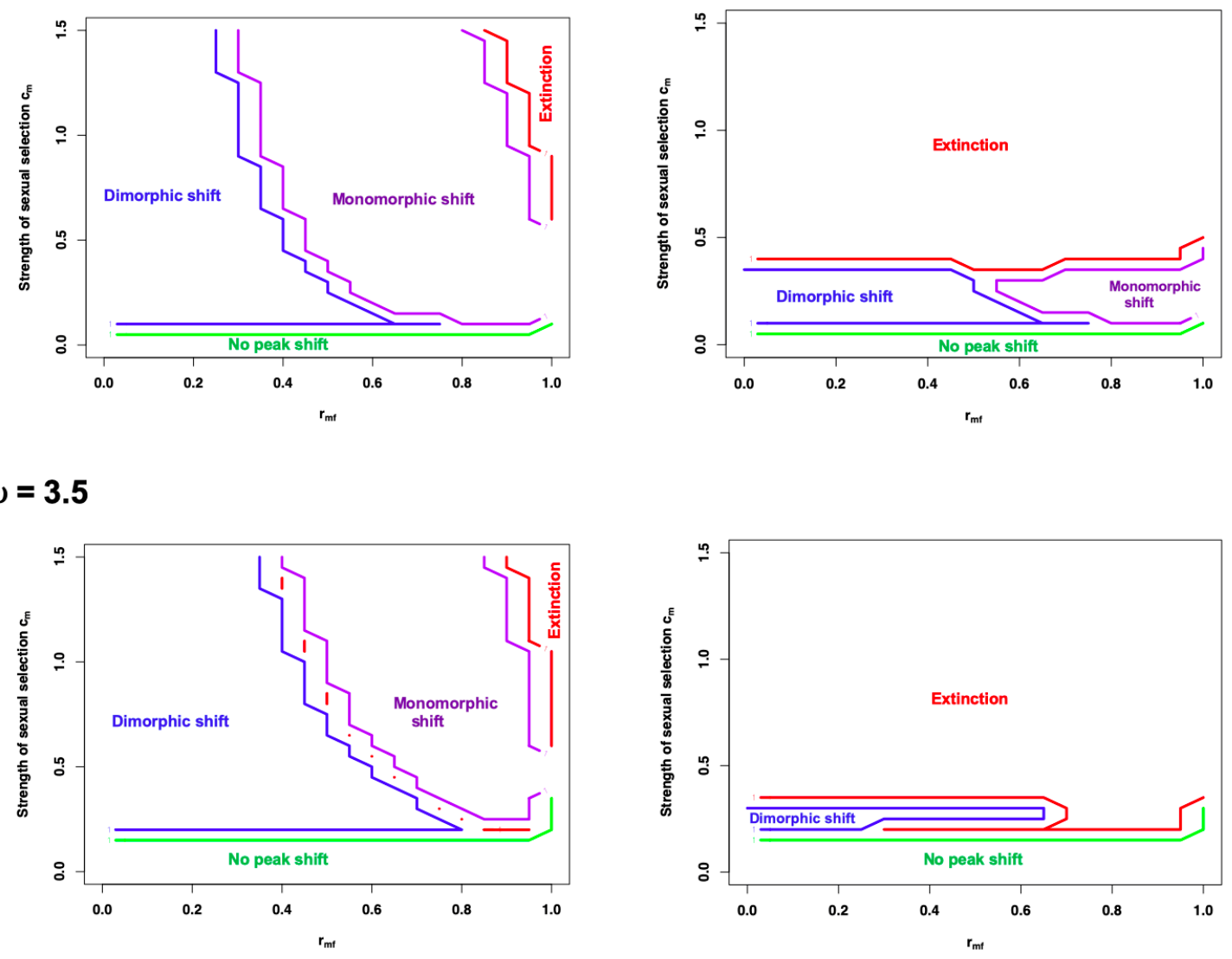

$\mathbf{C} \omega=2$
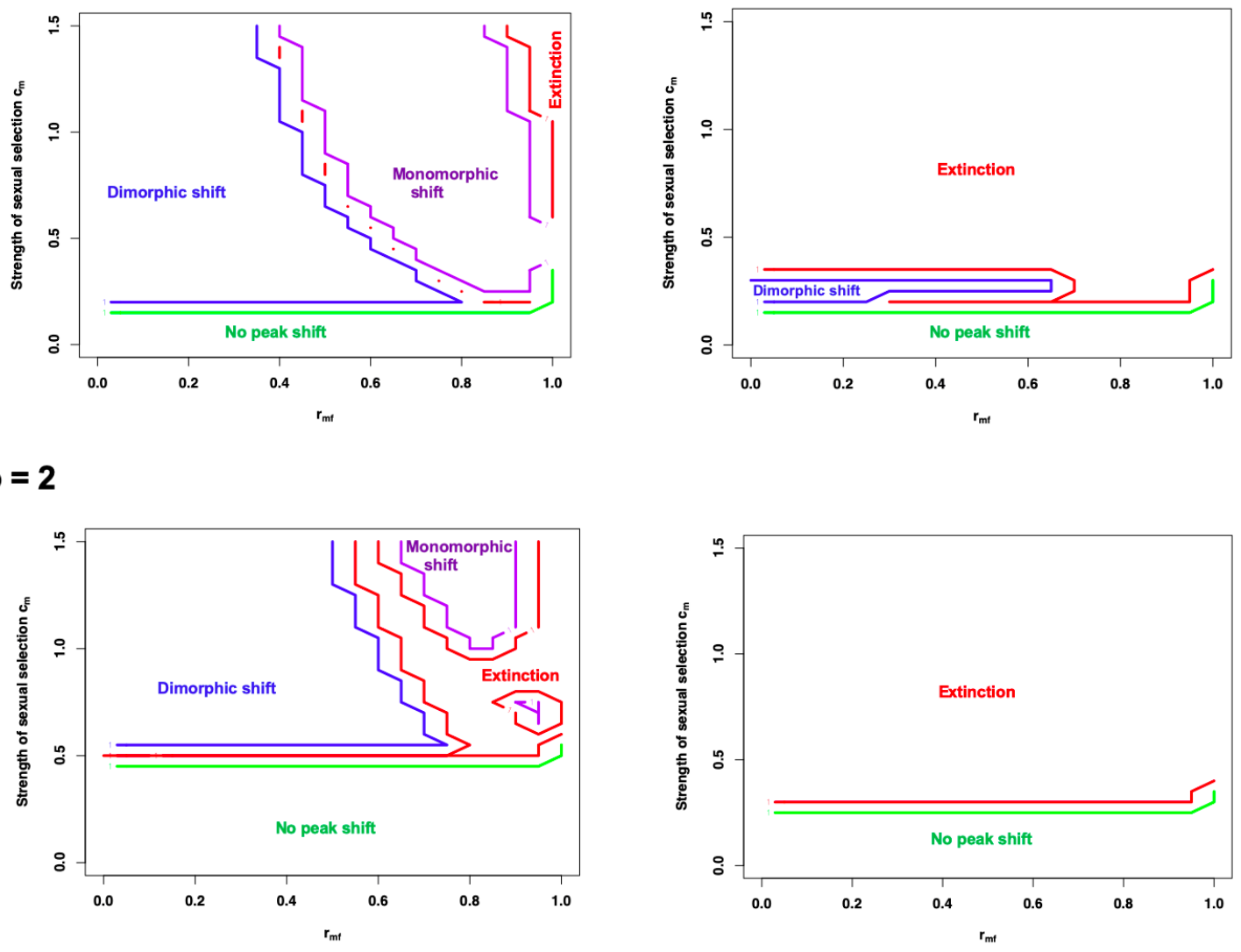

279 Figure 2. Deterministic outcomes as a function of sexual selection and $\boldsymbol{r}_{\mathrm{mf}}$. Panels show deterministic (i.e. no drift) outcomes of 1000 generations of evolution, calculated

281 numerically, corresponding to three different strengths of stabilizing selection. Left panels 282 assume female demographic dominance, right panels assume male and female fitness both 
determine population growth rate. 'Dimorphic shift' refers to a male-only peak shift,

284 'Monomorphic shift' refers to a peak shift in both sexes (illustrated in Fig 2A). No peak shift

285 means the lineage remains in the vicinity of the ancestral optimum. Small gaps between

286 regions represent plotting limitations of overlaid contour plots. 
309 1. Fisher RA. The genetical theory of natural selection. Oxford: The Clarendon press; 1930. xiv, $272 \mathrm{p} \mathrm{p}$.

311 2. Frank SA. Wright's Adaptive Landscape Versus Fisher's Fundamental Theorem. In:

312 Svensson EI, Calsbeek R, editors. The Adaptive Landscape in Evolutionary Biology: Oxford 313 University Press; 2013.

$3143 . \quad$ Wright S. The roles of mutation, inbreeding, crossbreeding and selection in evolution.

315 Proceedings of the Sixth Annual Conference of Genetics. 1932;1:356-66.

316 4. Coyne JA, Orr HA. Speciation. Sunderland, MA: Sinaur; 2004.

317 5. Coyne JA, Barton NH, Turelli M. Perspective: a critique of Sewall Wright's shifting 318 balance theory of evolution. Evolution. 1997;51:643-71.

319 6. Goodnight CJ. Wright's Shifting Balance Theory and Factors Affecting the

320 Probability of Peak Shifts. In: Svensson EI, Calsbeek R, editors. The Adaptive Landscape in

321 Evolutionary Biology: Oxford University Press; 2013.

322 7. Lande R. Expected time for random drift of a population between stable phenotypic 323 states. Proceedings of the National Academy of Sciences. 1985;82:7641-5.

324 8. Barton NH, Charlesworth B. Genetic revolutions, founder effects and speciation.

325 Annual Review of Ecology and Systematics. 1984;15:133-64.

3269 9rice T, Turelli M, Slatkin M. Peak shifts produced by correlated response to

327 selection. Evolution. 1993;47(1):280-90.

328 10. Uyeda JC, Hansen TF, Arnold SJ, Pienaar J. The million year wait for 329 macroevolutionary bursts. Proceedings of the National Academy of Sciences of the United 330 States of America. 2011;108:15908-13.

331 11. Gingerich PD. Rates of evolution on the time scale of the evolutionary process.

332 Genetica. 2001;112:127-44. 
12. Lande R. Sexual dimorphism, sexual selection, and adaptation in polygenic characters. Evolution. 1980;34(2):292-305.

335 13. Lande R, Kirkpatrick M. Ecological speciation by sexual selection. Journal of 336 Theoretical Biology. 1988;133:85-98.

337 14. Bonduriansky R. Sexual selection and sexual conflict as engines of ecological 338 diversification. American Naturalist. 2011;178:729-45.

339 15. Felsenstein J. Excursions along the interface between disruptive and stabalizing 340 seleciton. Genetics. 1979;93:773-95.

341 16. Arnold SJ, Pfrender ME, Jones AG. The adaptive landscape as a conceptual bridge

342 between micro- and macroevolution. Genetica. 2001;112/113:9-32.

343 17. Lande R. Natural selection and random genetic drift in phenotypic evolution.

344 Evolution. 1976;30:314-34.

345 18. Wright S. Evolution in Mendelian populations. Genetics. 1931;16:97-159.

346 19. Matthews G, Hangartner S, Chapple DG, Connallon T. Quantifying maladaptation 347 during the evolution of sexual dimorphism. Proceedings of the Royal Society of London 348 Series B. 2019;286:20191372.

349 20. De Lisle SP, Punzalan D, Rollinson N, Rowe L. Extinction and the temporal 350 distribution of macroevolutionary bursts. Journal of Evolutionary Biology. 2020;in press.

351 21. Cox RM, Skelly SL, John-Alder HB. A comparative test of adaptive hypotheses for 352 sexual size dimorphism in lizards. Evolution. 2003;57:1653-69.

353 22. Shine R. Ecological causes for the evolution of sexual dimorphism: a review of the 354 evidence. Quarterly Review of Biology. 1989;64(4):419-61.

355 23. Cox RM, Calsbeek R. Sexually antagonistic selection, sexual dimorphism, and the 356 resolution of intralocus sexual conflict. American Naturalist. 2009;173(2):176-87. 
24. Nali RC, Zamudio KR, Haddad CFB, Prado CPA. Size-dependent selective American Naturalist. 2014;184:727-40.

25. Janicke T, Häderer IK, Lajeunesse MJ, Anthes N. Darwinian sex roles confirmed across the animal kingdom. Science Advances. 2016;2:e1500983.

26. Poissant J, Wilson AJ, Coltman DW. Sex-specific genetic variance and the evolution of sexual dimorphism: a systematic review of cross-sex genetic correlations. Evolution. 2009;64(1):97-107.

27. Bonduriansky R, Rowe L. Sexual selection, genetic architecture, and the condition dependence of body size and shape in the sexually dimorphic fiy Prochyliz xanthostoma (Diptera: Piophilidae). Evolution. 2005(59):138-51. Press; 1960. 490 p.

29. Fairbairn DJ. Allometry for sexual size dimorphism: Pattern and process in the coevolution of body size in males and females. Annual Review of Ecology and Systematics. 1997;28:659-78.

30. Stephens PR, Wiens JJ. Evolution of sexual size dimorphisms in emydid turtles: ecological dimorphism, Rensch's rule, and sympatric divergence. Evolution. 2009;63:910-25. 31. Colwell RK. Rensch's rule crosses the line: Convergent allometry of sexual size dimorphism in hummingbirds and flower mites. American Naturalist. 2000;156(5):495-510. 


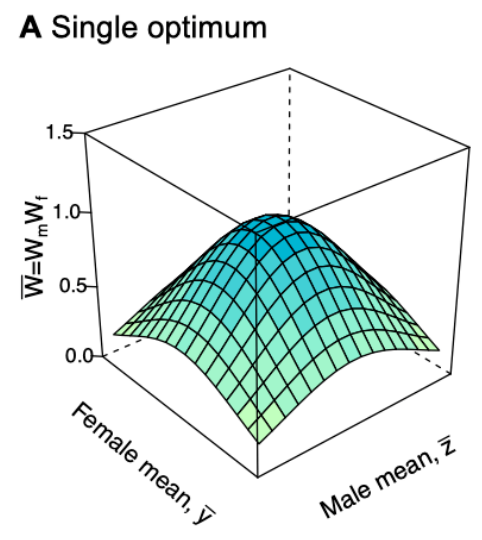

B Twin Peaks in one sex

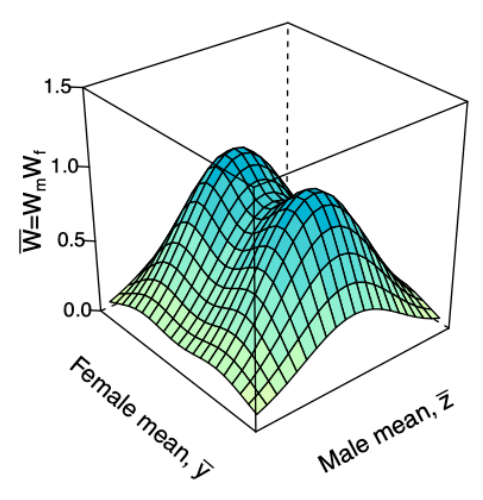

C Two-sex Twin Peaks

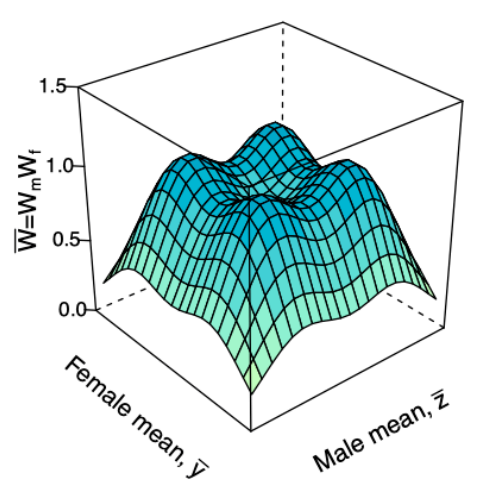

D The sexual selection load

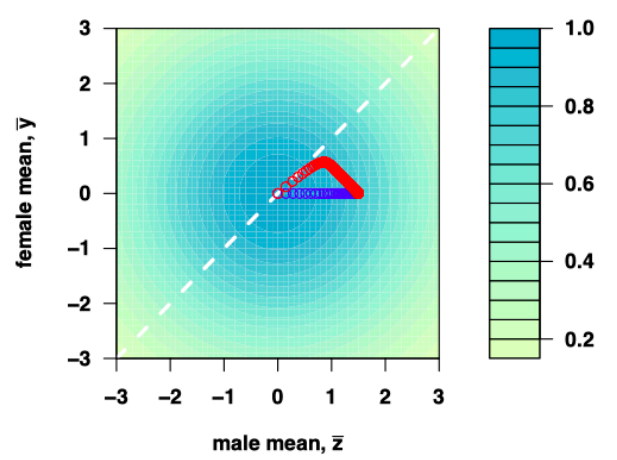

Figure S1. Models of stabilizing selection in two sexes. Panels illustrate the adaptive landscape, the function $\bar{W}_{m} \bar{W}_{f}$. In Lande's (1980) original model of evolution of sexual dimorphism by natural and sexual selection, a single multivariate optimum, corresponding to one optimum for the male trait and one for the female trait, is assumed (A). The 'Twin Peaks' model extends this to consider two optima in one trait (sex), with only a single optimum for the other trait (sex); (B). Extending the Twin Peak model to both sexes results in two optima for each trait, or four multivariate optima on the two-sex adaptive landscape $(\mathbf{C})$. As parameterized in this manuscript, the Two-sex Twin Peak model contains two sexually 
monomorphic optima, and two sexually dimorphic optima. Of interest is when and how peak

396 shifts from an ancestral monomorphic optimum occur. In this model sexual selection leads to

397 a displacement of males from their optimum $(\mathbf{C})$, the magnitude of which is proportional to

398 the strength of stabilizing natural selection. Two trajectories are shown starting from the

399 optimum; one where $r_{\mathrm{mf}}=0$ (blue) and one where $r_{\mathrm{mf}}=0.9$ (blue).

400

401

402

403

404

405

406

407

408

409

410

411

412

413 
A

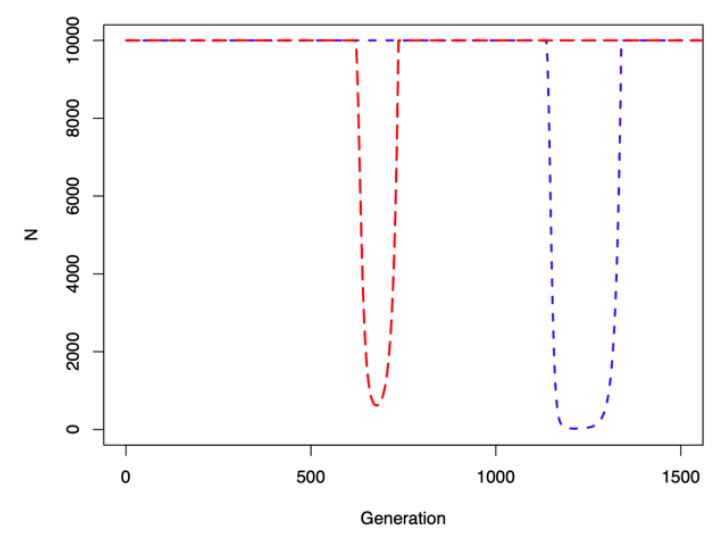

B

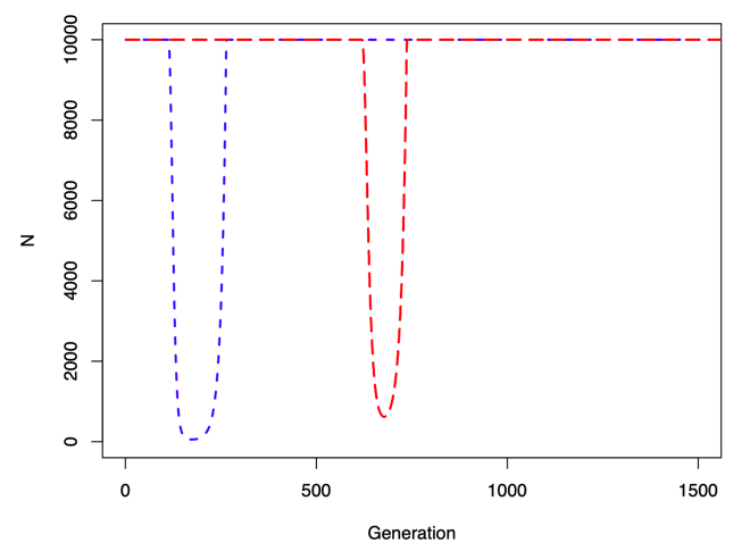

415 Figure S2 Demographic cost of valley crossing. Figures show population size from

416 trajectories plotted in Figure 1A and 1C. Panel A contrasts two values of the cross-sex

417 genetic correlation, $r_{\mathrm{mf}}=0.8$ (red line) or $r_{\mathrm{mf}}=0.9$ (blue line). Panel B contrasts two values

418 of the strength of sexual selection, $\mathrm{cm}=0.2$ (red line) or $\mathrm{cm}=0.3$ (blue line). Demographic

419 costs of a monomorphic peak shift, corresponding to the blue line in both panels, are higher

420 than for a dimorphic (male-only peak shift). Other parameter values are as described in

$421 \quad$ Figure 1.

422

423

424

425

426

427 

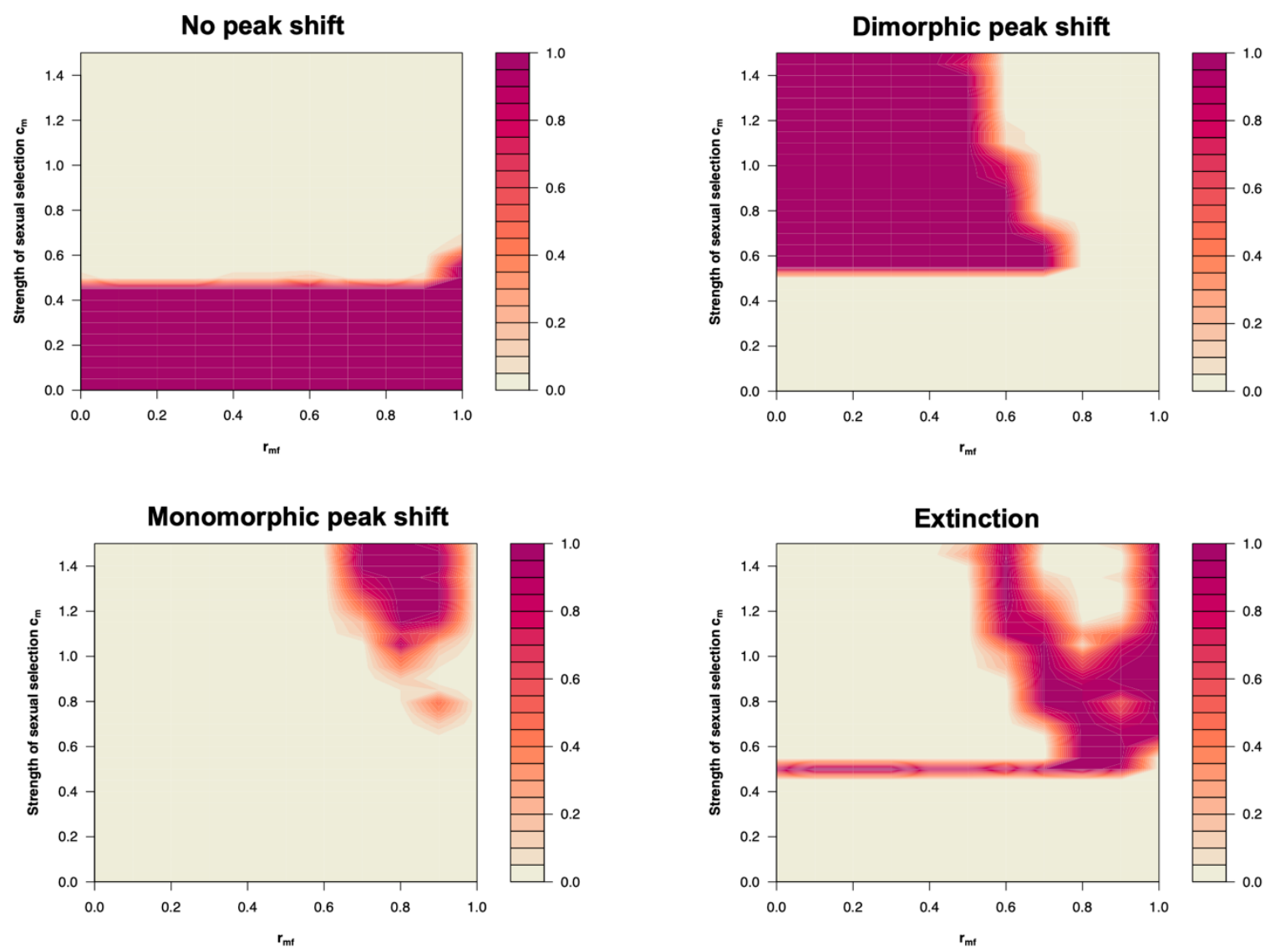

429 Figure S3 Stochastic outcomes as a function of sexual selection and $\boldsymbol{r}_{\mathrm{mf}}$. Panels show

430 stochastic (i.e., evolution under both selection and drift) outcomes of 1000 generations of

431 evolution, calculated numerically, corresponding to the scenario of strong natural selection

432 and female demographic dominance illustrated in Figure 2C. Thus, qualitative conclusions

433 on peak shifts remain similar to the deterministic case, and this holds for other parameter

434 values shown in Figure 2. 